\title{
Summary
}




\section{SUMMARY PAPER}

\section{SIDNEY VAN DEN BERGH}

Dominion Astrophysical Observatory, 5071 West Saanich Rd., Victoria, BC V8X 4M6 Canada

\section{INTRODUCTION}

Commission 50 of the IAU, which was the main sponsor of this Colloquium, has two functions:

1. helping astronomers find good new observing sites, and

2. protection of existing observatories.

Two years ago, Commission 50 sponsored a meeting in Flagstaff, AZ, U.S.A., devoted to "seeing" and site selection. The current meeting set out to study the three main threats to astronomical observation: light pollution, radio interference, and space debris.

Light pollution and radio interference are both part of the "electromagnetic fog" that threatens to blind us as we search for dim signals from the distant parts of the Universe and, perhaps, from intelligent life in other parts of the galaxy.

\section{LIGHT POLLUTION}

At this meeting, we have heard how some observatories, like Mt. Wilson, have been overwhelmed by light pollution, Others, such as Kitt Peak and Palomar, are waging successful battles that will delay the time when they too will be blinded forever. They are fighting light pollution rather than running away from it! Experience at a number of observatories shows that the battle against light pollution is a time consuming one that requires a major fraction of the time and energy of at least one staff astronomer, e.g. Crawford at Kitt Peak, Brucato at Palomar, Bolton at the David Dunlap Observatory, etc. I think it is fair to say that we have developed a consensus in favor of the idea that major observatories should vigorously support such activities. Perhaps the most encouraging news that we have heard at this meeting is that low pressure sodium lighting, which concentrates almost all of its energy into a single narrow wavelength bands, is gaining wide acceptance. This is partly due to our efforts, but more importantly, to the fact that it is presently more cost effective than other types of outdoor illumination. Unfortunately, other forms of lighting, that are potentially more harmful to astronomy, are showing considerable promise in the laboratory. In particular, metal halide head lights for cars constitute a possible future threat to 
optical astronomy.

Hunter and Crawford estimate that the light which is wasted by shining upwards towards the stars costs in excess of one billion dollars per year in the U.S.A. It is a sobering thought that this greatly exceeds the amount that U.S.A. taxpayers spend each year to support astronomy. In the same vein, one might note that about $1 \times 10^{3}$ people are presently working full time in the U.S.A. on tracking space debris, a figure that is comparable to the number of active optical astronomers in America.

\section{RADIO INTERFERENCE}

Govin Swarup has reminded us of the fact that research on radio radiation at metre wavelengths represents a race against time. Ten years ago we did not have the technology to undertake these studies at high sensitivity, and a decade from now the interference from Earth-based transmitters, will, in most countries, be too intense to continue such investigations. Clearly, we must hasten to exploit this window of opportunity. It has sometimes been suggested that radio astronomers could avoid interference problems by going to the back side of the Moon, But, as David Crawford remarked somewhat cynically: "The polluters are better financed than we are, so they will get there first."

A particular severe problem has recently developed due to emission by the GLONASS navigational satellite system. John Galt reported that these emissions presently destroy about $40 \%$ of his observations of the $1612 \mathrm{MHz}$ line of hydroxyl. When all 24 satellites of this system are operational, the $1612 \mathrm{MHz}$ window on the Universe will have become permanently closed to mankind. Clearly, we need prior consultation and international agreements to prevent future repetition of such an environmental misfortune. At this meeting, Vern Pankonin and Tom Gergely have emphasized the fact that astronomers need to remain vigilant to make sure that other important radio frequency windows remain open.

\section{SPACE DEBRIS}

Thirty years ago, it was widely believed that the oceans were so large that man could not possibly pollute them. Lubos Perek has reminded us that we are in danger of repeating this mistake by assuming that the ocean of space is so vast that it will always retain its pristine purity. During the three decades that have elapsed since the space age began, the amount of matter within $2000 \mathrm{~km}$ of the Earth's surface has increased by a factor of 10,000 . Fortunately, most of this material is presently still in relatively large units. The number of large objects near the Earth is, however, doubling every decade. As a result, the number of hypervelocity collisions, which produce numerous small fragments, is beginning to increase exponentially. This cascading effect will, in about a century, produce a lethal "asteroid shell" around the Earth. According to Don Kessler, the collision rate will go critical once the amount of matter in near-Earth orbit increases to between 10 and 100 times its present value. Once the collision rate goes critical, this runaway process can no longer be stopped by cutting off the 
injection of rockets into orbit. As a result, all operations in near-Earth and in geostationary orbit will become impossible, On a historical timescale, the Space Age is likely to be no more than a delta function that will last for only a few generations.

Back of the envelope calculations indicate that the illumination by sunlight of the pulverized space debris might turn night into permanent twilight. Detailed calculations using realistic particle fragmentation spectra and taking the Poynting-Robinson effect into account will, however, be required to firm up this tentative suggestion.

We do not yet know if it is already too late to prevent the formation of a terrestrial asteroid shell. Such an environmental catastrophe would transform near-Earth space into a brightly lit killing-field in which neither satellites nor astronauts could long survive. 